\title{
Fracture of the Styloid Process in an Elderly Patient Caused by Rolling Over During Sleep: A Case Report
}

\author{
Hirofumi Namiki ${ }^{\mathrm{a}, \mathrm{c}}$, Tadashi Kobayashi ${ }^{\mathrm{b}}$
}

\begin{abstract}
Fracture of the styloid process (SP) of the temporal bone is a rare traumatic injury in normal patients. The present report describes a fracture of the SP in an elderly patient caused by rolling over during sleep. A 67-year-old Japanese woman presented with sudden neck pain that had occurred upon rolling over in bed the previous night. A computed tomography scan revealed a fracture of the SP. She recovered with conservative treatment. Clinicians should consider a fracture when an elderly patient develops sudden neck pain by rolling over.
\end{abstract}

Keywords: Head injury; Aged; Japan; Rural population

\section{Introduction}

The styloid process (SP) of the temporal bone is a cylindrical projection on the inferior aspect of the petrous bone. Fracture of an elongated SP is more common [1] than fracture of a normally sized SP, which is a rare event [2]. Previous reports have shown that facial or neck trauma is the most frequent cause of fracture of the SP [3].

No reports have indicated that rolling over is a cause of fracture of the SP. We herein describe a patient who developed a fracture of the SP associated with sudden neck pain by rolling over during sleep. Thus, fracture of the SP should be considered when elderly patients develop sudden neck pain after a casual motion such as rolling over.

\section{Case Report}

A 67-year-old woman developed a sudden onset of severe left-

Manuscript accepted for publication January 11, 2017

aYonaguni Municipal Clinic, Japan Association for Development of Community Medicine, 125-1 Yonaguni, Yonaguni-cho, Yaeyama-gun, Okinawa-ken 907-1801, Japan

bepartment of General Medicine, Hirosaki University School of Medicine \& Hospital, 53 Hon-cho, Hirosaki-shi, Aomori-ken 036-8563, Japan

${ }^{\mathrm{c} C}$ Corresponding Author: Hirofumi Namiki, Yonaguni Municipal Clinic, Japan Association for Development of Community Medicine, 125-1 Yonaguni, Yonaguni-cho, Yaeyama-gun, Okinawa-ken 907-1801, Japan.

Email: japan_hiro2000@yahoo.co.jp

doi: https://doi.org/10.14740/jmc2741w sided neck pain the night before presentation. She presented 6 $\mathrm{h}$ after the onset of the symptom. Her medical history included diabetes and osteoporosis. There was no family history of bone disease. Her regular medications were drugs for diabetes and osteoporosis. The patient's chief complaint was pain on the left side of her neck. She experienced worsening pain and no limitation during her jaw movement. She reported no surgical history or tonsillectomy. The patient did not complain of symptoms mimicking glossopharyngeal neuralgia. Upon examination, she had severe neck pain but normal vital signs. Neck examination revealed tenderness in near front of the mastoid process. Other examinations were normal. Blood test and X-ray findings were also normal. Because of concern regarding an underlying fracture, a computed tomography scan of the neck was performed, which revealed a fracture of the SP (Fig. 1).

Thus, the patient was diagnosed with a fracture of the SP and received conservative treatment and follow-up. A neck brace was placed to limit her neck movement, and an analgesic was prescribed (acetaminophen, $500 \mathrm{mg}$ every $8 \mathrm{~h}$ for 8 weeks). After 2 months when conservative treatment started, her neck pain gradually resolved.

\section{Discussion}

Investigations of the epidemiology of fracture of the SP have indicated that such fractures are rare traumatic events that frequently occur in association with other head and neck fractures, especially mandibular fractures [1]. Several reports have described fractures of the SP in patients with Eagle syndrome [3-5]. However, no reports have described fracture of the SP due to a minor trauma such as that induced by rolling over.

With respect to the cause of the fracture of the SP in the patient, traumatic injury is likely. When she rolled over, her neck sprained unnaturally. She also had a history of osteoporosis; thus, bone fragility might have contributed to the fracture. She had no other predisposing risk factors for fracture of the $\mathrm{SP}$, although the influence of osteoporosis was not completely ruled out. Therefore, the cause of the fracture of the SP in her could be considered to be related to rolling over.

Regarding the therapeutic approach to the fracture of the $\mathrm{SP}$ in the patient, conservative management was chosen and provided good recovery. However, optimal management of a fracture of the SP depends on its severity and displacement [3]. Surgical removal of an SP bone chip could be necessary in some patients, but conservative treatment might be sufficient in those with only mild discomfort. The patient was treated conservatively with a neck brace to limit her neck movement, 


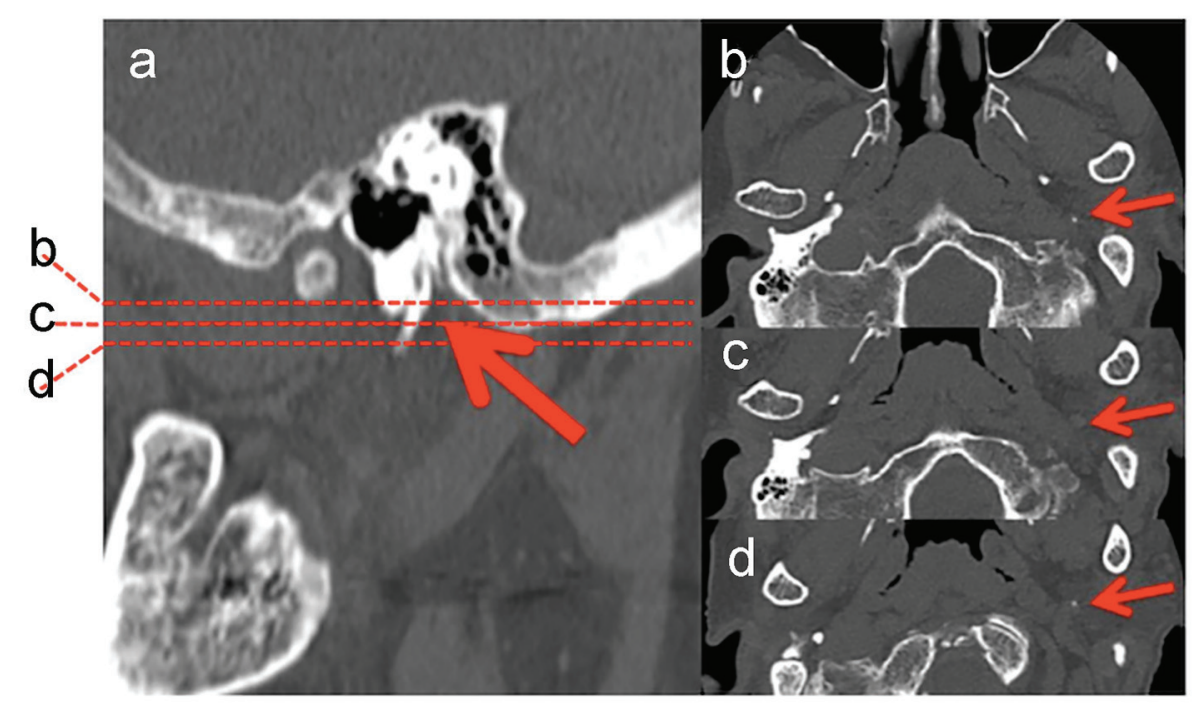

Figure 1. Computed tomography of the neck showing a fracture of the styloid process. Red arrows show the styloid process and the fracture. (a) Sagittal view. (b-d) Coronal view on each red dotted line of (a).

and we prescribed an analgesic. To the best of my knowledge, no reports have described fractures in elderly patients in association with rolling over. Fracture of the styloid process should be considered in elderly patients with sudden onset of neck pain during sleep.

\section{References}

1. Reichart PA, Sooss W. Fracture of the styloid process of the temporal bone: an unusual complication of dental treatment. Report of a case. Oral Surg Oral Med Oral Pathol. 1976;42(2):150-154.
2. Kermani H, Dehghani N, Aghdashi F, Esmaeelinejad M. Nonsyndromic Isolated Temporal Bone Styloid Process Fracture. Trauma Mon. 2016;21(1):e24395.

3. Blythe JN, Matthews NS, Connor S. Eagle's syndrome after fracture of the elongated styloid process. Br J Oral Maxillofac Surg. 2009;47(3):233-235.

4. Orlik K, Griffin GD, Zoumberakis E. Unilateral neck pain: a case of Eagle syndrome with associated nontraumatic styloid fracture. Am J Emerg Med. 2014;32(1):112 e111-112.

5. McCorkell SJ. Fractures of the styloid process and stylohyoid ligament: an uncommon injury. J Trauma. 1985;25(10):1010-1012. 